\title{
Prevalence and Pattern of Ocular Involvement in Patients Attending Alexandria University Rheumatology Clinic: A Pilot Study
}

\author{
Tayel MY' Mohannad N ${ }^{1}$, El Gerby AH ${ }^{1}$, Amin RM² \\ Departments of Internal Medicine, Rheumatology Unit ${ }^{1}$, \\ Ophthalmology, Uveitis and Ocular Immunology Division²; Alexandria University; Egypt
}

\begin{abstract}
It is known that patients with rheumatic diseases can show various ocular manifestations. Ocular involvement is well known in most rheumatic diseases including rheumatoid arthritis, systemic lupus erythematosus, ankylosing spondylitis, Behçet disease, anti-phospholipid syndrome, reactive arthritis and others. Their clinical expression and outcome vary between different populations. Although there are epidemiological studies on uveitis from different geographic areas, registry of patterns of eye affection among rheumatic patients in Egypt is highly deficient. Objective: To identify the prevalence and characteristics of uveitis and other patterns of immune eye involvement in patients referred to Alexandria University outpatient rheumatology clinic during the period from January 2013 to December 2013. Methods: A cross sectional analysis of a cohort of 1500 patients with immune diseases attending the outpatient rheumatology clinic was done. Of these, 100 patients had relevant eye complaints. The demographic features, clinical presentations, patterns of eye involvement, and modes of treatment of eye disease were registered for all patients. Results: 59\% of the patients were female. Mean age was 39.26 years and the mean duration of systemic disease was 41 months. Mean duration of eye disease was 30 months. Behcet's disease was the most common identifiable cause of uveitis (24\%) followed by JIA associated uveitis $(6 \%)$ and VKH (5\%). Idiopathic anterior uveitis was the most common uveitic entity representing $32 \%$ of cases. $14 \%$ needed intraocular drug injections. Topical as well as systemic steroids were added in 58\%, synthetic or biological DMARDs in 14\%, and surgery in 14\%. Conclusions: Ocular complications are common among rheumatic patients. Awareness of the possibility of serious eye involvement should always be kept in mind. Routine ophthalmological evaluation of all patients affected with any rheumatic disease is mandatory \& should be stressed upon in all guidelines \& recommendations in rheumatology. [Egypt J Rheumatology \& Clinical Immunology, 2015; 3(2): 105-110]
\end{abstract}

Key Words: Eye, Rheumatic disease, uveitis.

\section{INTRODUCTION}

Uveitis describes a group of intraocular inflammatory disorders. The uvea comprises the iris, ciliary body, and choroid. Uveitis may affect other tissues in the eye, however, including the retina (uveoretinitis), retinal blood vessels (retinal vasculitis), the vitreous (vitritis), and the optic nerve (papillitis). Therefore, uveitis is anatomically classified inanterior, intermediate, posterior and panuveitis ${ }^{1}$. The cause of uveitis may be autoimmune, infection related, drug induced, traumatic, or associated with malignancy (when it is known as the masquerade syndrome). About half of cases are idiopathic and are presumed to be autoimmune. ${ }^{2}$

Immune-mediated ocular inflammation causes severe debilitation and visual loss. ${ }^{3}$

Unfortunately, not all patients with rheumatic diseases follow up their condition with a rheumatologist; moreover, many rheumatologists often overlook the eye.

Almost all the anatomical parts of the eye could be a target for an immunological reaction depending upon the underlying etiological disease. ${ }^{4}$ Inflammation of ocular structures can lead to secondary complications including cataract, glaucoma, and optic atrophy. ${ }^{4,5}$ Many ocular complications are indicators of active systemic disease process and some of them are markers of severe and potentially life-threatening systemic involvement. ${ }^{6}$

A patient with RA who develops peripheral ulcerative keratitis, often associated with scleritis, is at risk of a sight as well as life threatening vasculitis and this finding heralds the need for further immunosuppressive measures for control of a severely active systemic disease. ${ }^{7,8}$

The pattern and prevalence of uveitis is largely influenced by a multitude of factors, including ethnic, 
genetic, environmental factors, referral and reporting bias. $^{9-14}$

Although there are some epidemiological studies on uveitis from different geographic areas and populations, data from Africa is generally scarce and from North Africa is almost lacking. Such data will have significant clinical and research applications and would help plan appropriate treatment protocols on community basis.

In this study, we analyze the causes of uveitis in a tertiary rheumatology referral center in North Egypt in patients reporting eye problems during routine review of systems over the period from January 2013 to December 2013.

\section{PATIENTS AND METHODS}

This cross sectional study was carried out on data from consecutive patients who were seen in a tertiary referral rheumatology clinic in Alexandria Main University Hospital, Alexandria, Egypt in 2013. Information regarding age, sex, and race was recorded. Details on ocular and systemic complaints, examination, and investigations were recorded for all patients. Ocular examination included slit lamp examination, tonometry, fundus biomicroscopy, and indirect ophthalmoscopy. Ultrasonography, optical coherence tomography, and fundus fluorescein angiography were ordered when needed.

1500 patients were recruited to this study, of which 100 patients had relevant eye complaints. They were classified according to the type of the underlying disease. All patients were questioned about systemic disease duration, age at onset, duration and pattern of eye involvement (unilateral or bilateral, course: acute, chronic or recurrent), type and dose of medications (topical, systemic or both), eye complications, and the need for surgery. Local eye examination was performed to determine the type and location of eye affection (anterior or posterior segment, active or not, associated corneal or retinal involvement). Data analysis was performed using (SPSS Inc, Chicago, USA), and $\mathrm{P}$ value $\leq 0.05$ was considered to be statistically significant.

\section{RESULTS}

A total of 100 patients (169 eyes) were included. The mean age at onset of uveitis was 39.26 (range 7-70) years, and the male-to-female ratio was 0.69 . In our study, all patients were native Egyptians mainly from North Egypt governorates. Ocular involvement was unilateral in 31 patients. The mean duration of systemic disease was 41 months and 30 months for eye disease. The demographic data and patterns of eye involvement are demonstrated in Table (1) and Figures (1 and 2) respectively.

Anterior uveitis (AU) was most common $(27 \%)$, followed by panuveitis $(20 \%)$, posterior uveitis (28\%), and intermediate uveitis (3\%). Other forms of ocular immune disorders visiting the clinic included ocular cicatricial pemphigoid and Sjogren's syndrome in 4 patients.

The most common causes of uveitis regardless of the anatomical forms were Behçet disease (BD) (24\%); juvenile idiopathic arthritis (JIA) associated uveitis (6\%), Vogt-Koyanagi-Harada (VKH) syndrome (5\%) and sarcoidosis (4\%). Retinal vasculitis was found in $20 \%$. The most common complications were cataract $(21.6 \%)$, ocular hypertension (12\%) and macular edema (5.6\%).

We reported one patient with each psoriatic arthritis, PAPS, MCTD, granulomatous uveitis, 1ry Sjogren syndrome, systemic sclerosis, and one with combined Wegner granulomatosis and SLE. Diseases with corresponding pattern of eye affection are demonstrated in Table (2). Eighty two per cent of patients had the rheumatic disease and eye affection simultaneously. $16 \%$ of patients had the ocular involvement following their systemic disease diagnosis, and of note, was the fact that the onset of eye involvement after systemic disease onset was long \& reached up to 180 months in 16 $\%$ of patients. Eye involvement before systemic disease occurred in 2 patients, both with RA (24 months and 12 months before RA onset respectively).

Table (3) shows the mode of treatment of eye affection. Most patients needed combined therapy; intra-ocular, topical as well as systemic steroids together with other synthetic or biological DMARDs. 
Table 1. Demographic data of the patients.

\begin{tabular}{lcc}
\hline Gender & Female & No (\%) \\
& Males & $59(59)$ \\
Age in yrs & Min-max (Mean) & $41(41)$ \\
Disease duration (ms) & Min-max (Mean) & $7-70(39.26)$ \\
Eye disease duration (ms) & Min-max (Mean) & $0-264(41)$ \\
Eye involvement in relation & to disease onset & $0-180(30)$ \\
& Before & $2(2 \%)$ \\
& After & $16(16 \%)$ \\
& At the same time & $82(82 \%)$ \\
\hline
\end{tabular}

Table 2. Diseases with corresponding pattern of eye affection.

\begin{tabular}{|c|c|c|c|c|c|c|c|c|}
\hline Disease & $\begin{array}{c}\text { No of } \\
\text { patients }\end{array}$ & Anterior & Intermediate & Posterior & Panuveitis & Corneal & Retinal & Sogren's \\
\hline $\mathrm{AI}$ & 32 & 19 & 2 & 6 & 2 & 3 & 0 & 0 \\
\hline Behcet ds & 24 & 2 & 1 & 11 & 9 & 0 & 1 & 0 \\
\hline RA & 10 & 5 & 0 & 1 & 0 & 2 & 1 & 1 \\
\hline JIA & 6 & 2 & 0 & 2 & 2 & 0 & 0 & 0 \\
\hline V K H & 5 & & & & & & & \\
\hline AS & 3 & 3 & 0 & 0 & 0 & 0 & 0 & 0 \\
\hline Sarcoidosis & 4 & 1 & 0 & 0 & 2 & 0 & 0 & 1 \\
\hline SLE/APS & 4 & 0 & 0 & 4 & 0 & 0 & 0 & 0 \\
\hline SLE & 3 & 0 & 0 & 0 & 1 & 0 & 2 & 0 \\
\hline OCP & 2 & & & & & & & \\
\hline others & 7 & 2 & 0 & 1 & 2 & 0 & 1 & 1 \\
\hline Total & 100 & 34 & 3 & 25 & 18 & 5 & 5 & 3 \\
\hline
\end{tabular}

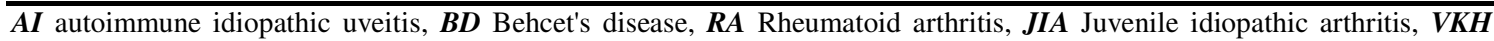
Vogt-Koyanagi-Harada (VKH) syndrome, $\boldsymbol{A S}$ ankylosing spondylitis, $\boldsymbol{S} \boldsymbol{L} \boldsymbol{E}$ Systemic lupus erythematosus, $\boldsymbol{A P S}$ antiphospholipid syndrome, $\boldsymbol{O C P}$ Ocular cicatricial pemphigoid.

Table 3. Type of treatment of eye diseases.

\begin{tabular}{|c|c|c|c|c|c|c|c|c|c|}
\hline \multirow{3}{*}{ IO } & \multirow{3}{*}{$\begin{array}{c}\text { Topical } \\
\text { Only }\end{array}$} & \multirow{3}{*}{ SDMARDs } & \multicolumn{4}{|c|}{ bDMARDs } & \multirow{3}{*}{ Steroids } & \multirow{3}{*}{ Surgery } & \multirow{3}{*}{ Others } \\
\hline & & & \multicolumn{4}{|c|}{14} & & & \\
\hline & & & Infliximab & Adalimumab & Etanercept & $\begin{array}{c}\text { tried } 2 \\
\text { biologics }\end{array}$ & & & \\
\hline 14 & 58 & 44 & 7 & 4 & 2 & 1 & 57 & 14 & 5 \\
\hline
\end{tabular}




\section{Pattern of eye involvement (type)}

- Unilateral Bilateral

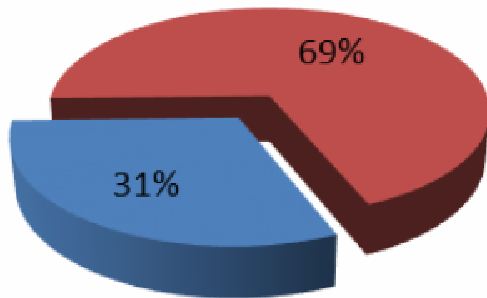

\section{Pattern of eye involvement (Onset)}

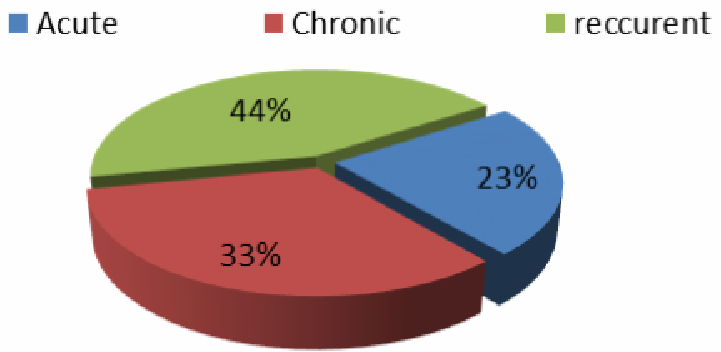

Figures 1 and 2. Pattern of eye involvement.

\section{DIscussion}

The magnitude of eye problems associating rheumatic diseases is not well estimated in some populations and data concerning its pattern is highly deficient. $^{3,6,11}$ Uveitis can occur either as a comanifestation of various autoimmune disorders; an extra-articular manifestation of these diseases or it can arise as a purely idiopathic ocular inflammation. It can affect any part of the eye from the cornea and sclera anteriorly to the retina and uveal tract posteriorly, and it occurrence determines the need for more aggressive immunosuppressive therapy. ${ }^{1,3,12,13}$ The incidence, severity, and disease course of uveitis will vary depending upon the natural history of the underlying systemic disease and the extent of the inflammatory process. In our study, the most common anatomic diagnosis was anterior uveitis. Behcet's disease was the most common identifiable cause of uveitis followed by JIA associated uveitis and VKH. Idiopathic AU was the most common uveitic entity.

An effect of referral and selection bias should be considered when looking into similar data, as most uveitis studies are published from tertiary referral centers causing perhaps an overrepresentation of more complicated severe forms of pan or posterior uveitis. Our study is perhaps affected by inclusion bias as the study population is drawn from an outpatient department.

Regarding the pattern \& associations of uveitis reported from other countries, it was noted that in a review of existing medical records of 5970 patients evaluated by theUveitis Service at the University of Illinois at Chicago between 1973 and 2007 with adiagnosis of chronic anterior uveitis (CAU); $31 \%$ carried a diagnosis of CAU.Idiopathic disease was diagnosed in $54 \%$ of patients, ocular sarcoidosis in $14 \%$, Fuchs heterochromic iridocyclitis (FHI) in $12 \%$ and JIA in $6 \%$ of patients. 
The frequency of diagnosis of idiopathic CAU decreased over time, with no significant change for sarcoidosis, FHI or JIA. An increase in frequency of diagnosis was observed for HLA-B27-relateddisease and uveitis related to multiple sclerosis and inflammatory bowel disease. It was concluded that the relative frequency of idiopathic disease has decreased over the past 35 yearsat their center. This may be related to an increase in the diagnosis of CAU associated with HLAB27positivity, inflammatory bowel disease (including family history) and multiple sclerosis.

Despite the advances over the last 35 years, idiopathic disease still comprises at least $39 \%$ of patients with CAU each year. ${ }^{14}$

While the characteristics of uveitis in a Canadian referral centre performed between September 2004 and March 2006, published in 2010, including Two hundred eighty-five patients (Three hundred sixtyfour eyes) with anterior uveitis, intermediate uveitis, or panuveitis concluded that according to the anatomical subtypes there were 313 anterior, 36 intermediate, and 15 panuveitis. Systemic associations included ankylosing spondylitis (11.3\%), inflammatory bowel disease $(6 \%)$, and sarcoidosis $(4.1 \%)$, and herpes infections (3.8\%). An HLA-B27related association was found in 86 patients $(23.6 \%)$. Patients with uveitis-associated complications or back symptomatology were more likely to have a recurrence. Of note, patients with posterior uveitis were excluded from this study. ${ }^{15}$

Whereas the anatomical and etiological spectrum of uveitis in an urban multi-ethnic population from Barcelona, Spainperformed between 1 January 2009 and 31 December 2012 after exclusion of after exogenous endophthalmitis, surgery-related, post-traumatic and toxic uveitis along with masquerade syndromes were investigated. From 1022 patients, 52\% were AU, $23 \%$ posterior, $15 \%$ panuveitis and $9 \%$ intermediate uveitis. Etiologically, $26 \%$ were unclassifiable, $29 \%$ infectious, $25 \%$ associated with systemic immune diseases, and $20 \%$ corresponded to ocular-specific syndromes. Among classified causes, herpesvirus $(12 \%)$, toxoplasma (7\%), BD (5\%), HLA-B27-isolated AU (5\%), ankylosing spondylitis (5\%), tuberculosis-related uveitis (TRU) (5\%), birdshot chorio retinopathy (3\%) and sarcoidosis (3\%) were the most frequent. Non-Spanish origin was recorded in $22 \%$, with $47 \%$ of VKH and $36 \%$ of toxoplasma cases coming from South America, $10 \%$ of BD and $11 \%$ of TRU fromAfrica and $24 \%$ of TRU cases from Asia. A mean annual incidence of 51.91 cases/100 000 inhabitants was found for the referral population. ${ }^{16}$
The patterns uveitis reported from Singapore demonstrated that $\mathrm{AU}$ was the predominant form of uveitis seen and most cases were unilateral (79.5\%) and idiopathic (50.4\%). Common etiological causes included FHI (5.6\%), ankylosing spondylitis (AS)related $\mathrm{AU}(5.1 \%)$, herpes simplex virus (HSV) $(4.7 \%)$, and herpes zoster virus (HZV) $(4.5 \%)$. There were increasing trends in AS-related $\mathrm{AU}$ from $3.2 \%$ in 2008 to $6.5 \%$ in 2010, and psoriasis-associated AU from $1.7 \%$ in 2005 to $4.0 \%$ in $2008 .^{17}$

Of the 42 patients with AU seen at a tertiary care eye hospital in south India, $14(33.33 \%)$ were HLAB27-positive and the remaining $28(66.66 \%)$ HLAB27-negative. Overall, 32 of the 42 had no causative association, three patients were diagnosed with JIA, two with ankylosing spondylitis, and two were associated with systemic tuberculosis, two with sarcoidosis and one with VKH syndrome. ${ }^{18}$

Data derived from Tunis showed thata total of 424 patients (596 eyes) were included. The mean age at onset of uveitis was 36 years, and the male-tofemale ratio was 0.66.Uveitis was unilateral in $56.4 \%$. AU was most common (48\%), followed by panuveitis (33.6\%), posterior uveitis (13.3\%), and intermediateuveitis $(5 \%)$. The most common causes were BD $(14.7 \%)$, toxoplasmosis $(10.2 \%)$, VKH syndrome (3.7\%) and sarcoidosis (3.3\%). Retinal vasculitis was found in $20 \%$. Behçet disease was the most common cause of chronic uveitis. The most common complications were cataract $(21.6 \%)$, ocular hypertension (12\%) and macular edema $(5.6 \%) .{ }^{19}$

Another recent study aimed to determine the pattern of childhood eye disorders in patients attendingoutpatient eye department of a rural eye hospital in central Ethiopia was published. It was concluded that the three most common causes of childhood ocular morbidity were conjunctivitis, ocularinjuries and refractive errors. However, these disorders require attention for propermanagement or early referral and 735 children were included; the idea of autoimmune uveitis was not addressed in their work. ${ }^{20}$

The same issue was raised in another study from Nigeria evaluating the reports of 286 children between January 2001 and December 2006, which concluded that the most common causes of childhood ocular morbidity were injuries, allergic conjunctivitis, infections and refractive errors omitting the fact that eye can be the window for detection of many systemic diseases. $^{21}$

Since uveitic entities follow different patterns in different regions and are influenced by a variety of demographic factors, epidemiologic studies can help improve our understanding of such diseases and provide appropriate diagnosis and timely management. ${ }^{6-11,22}$. 


\section{Conclusions}

Ocular complications are common among rheumatic patients in Egypt. Awareness of the possibility of serious eye involvement should always be kept in mind. Routine ophthalmological evaluation of all patients affected with any rheumatic disease is mandatory \& should be stressed upon in all guidelines $\&$ recommendations in rheumatology. Following a universal classification system and having populationbased studies from different countries, a more reliable data for comparison between different geographic areas can be provided.

We recommend extending this study to involve a large cohort of Egyptian patients with rheumatic diseases.

\section{REFERENCES}

1. GulyCM , Forrester JV. Investigation and management of uveitis. BMJ 2010 ; 341: 821-6

2. Rothova A, Buitenhuis HJ, Meenken C, Brinkman CJ, Linssen A, Alberts C, et al. Uveitis and systemic disease. Br J Ophthalmol 1992;76:137-41.

3. Durrani OM, Meads CA, Murray PI. Uveitis: a potentially blinding disease. Ophthalmologica 2004;218:223-36.)

4. Nussenblatt RB, Whitcup SM, Palestine AG. Uveitis: Fundamentals and Clinical Practice. 3rd ed. Philadelphia (PA): Mosby; 2004:58-68

5. AAO Basic and clinical Course Section 9: Intraocular inflammation and Uveitis, 2010-2011: 101.

6. Choudhary MM, Hajj-Ali RA, Lowder CY. Gender and ocular manifestations of connective tissue diseases and systemic vasculitides. Ophthalmol. 2014;2014:403042.

7. Mohsenin $\mathrm{A}^{1}$, Huang JJ. Ocular manifestations of systemic inflammatory diseases.Conn Med. 2012;76(9):533-44.

8. C. S. Foster, S. L. Forstot, and L. A. Wilson, "Mortality rate in rheumatoid arthritis patients developing necrotizing scleritis or peripheral ulcerative keratitis: effects of systemic immunosuppression," Ophthalmology. 1984; 91(10): 1253-63.

9. Henderly DE, Genstler AJ, Smith RE, Rao NA. Changing patterns of uveitis. Am J Ophthalmol 1987;103:131-6.
10. McCannel CA, Holland GN, Helm CJ, Cornell PJ, Winston JV, Rimmer TG. Causes of uveitis in the general practice of ophthalmology. UCLA Community-Based Uveitis Study Group. Am J Ophthalmol 1996;121:35-46.

11. Merrill PT, Kim J, Cox TA, Betor CC, McCallum RM, Jaffe GJ. Uveitis in the southeastern United States. Curr Eye Res 1997;16:865-74.

12. Kotake S, Furudate N, Sasamoto Y, Yoshikawa K, Goda C, Matsuda H . Characteristics of endogenous uveitis in Hokkaido, Japan. Graefes Arch ClinExpOphthalmol 1997;235:5-9.

13. Hamade IH, Elkum N, Tabbara KF. Causes of uveitis at a referral center in Saudi Arabia. OculImmunolInflamm 2009;17:11-6.

14. Birnbaum AD, Little DM, Tessler HH, Goldstein DA. Etiologies of Chronic Anterior Uveitis at a Tertiary Referral Center over 35 Years. OculImmunolInflamm. $2011 ; 19(1)$ : 19-25.

15. Chan SM, Gan KD, Weis E. Characteristics and predictors of recurrence of anterior and intermediate uveitis in a Canadian referral centre. Can J Ophthalmol2010;45(2):144-8.

16. Llorenç V, Mesquida M, Sainz de la Maza M, Keller J, Molins B, Espinosa G, Hernandez MV, Gonzalez-Martín J, Adán A. Epidemiology of uveitis in a Western urban multiethnic population. The challenge of globalization. ActaOphthalmol. $2015 ; 15$.

17. Tan WJ, Poh EW, Wong PY, Ho SL, Lim WK, Teoh SC. Trends in patterns of anterior uveitis in a tertiary institution in singapore. OculImmunolInflamm. 2013 Aug;21(4):270-5

18. Mathur G, Biswas J. Systemic associations of anterior uveitis in a tertiary care ophthalmic centre in south India. IntOphthalmol. 2012;32(5):417-21.

19. Chebil A, Baroudi B, Slim M, Chaker N, Lamloum M, Bouladi M, Houman H, El Matri L. Epidemiology of uveitis in the Tunis area. J FrOphtalmol. 2013 Nov;36(9):764-8.

20. MehariZA . Pattern of childhood ocular morbidity in rural eye hospital, Central Ethiopia. BMC Ophthalmology 2014, 14:50

21. Onakpoya OH, Adeoye AO. Childhood eye diseases in southwestern Nigeria: a tertiary hospital study. Clinics. 2009;64(10): 947-51.

22. Nashtaei EM, SoheilianM ,Herbort CP, Yaseri M. Patterns of uveitis in the middle East and Europe . J Ophthalmic Vis Res 2011; 6 (4): 233-40. 